\title{
ONLINE SUPERVISED LEARNING OF NON-UNDERSTANDING RECOVERY POLICIES
}

\author{
Dan Bohus, Brian Langner, Antoine Raux, Alan W Black, Maxine Eskenazi, Alex Rudnicky \\ \{dbohus, blangner, antoine, awb, $\max , a i r\} @ c s . c m u . e d u$ \\ Carnegie Mellon University, \\ Pittsburgh, PA, 15217
}

\begin{abstract}
Spoken dialog systems typically use a limited number of nonunderstanding recovery strategies and simple heuristic policies ${ }^{1}$ to engage them (e.g. first ask user to repeat, then give help, then transfer to an operator). We propose a supervised, online method for learning a non-understanding recovery policy over a large set of recovery strategies. The approach consists of two steps: first, we construct runtime estimates for the likelihood of success of each recovery strategy, and then we use these estimates to construct a policy. An experiment with a publicly available spoken dialog system shows that the learned policy produced a $12.5 \%$ relative improvement in the non-understanding recovery rate.
\end{abstract}

Index Terms - non-understandings, recovery strategy, recovery policy, spoken language interface

\section{INTRODUCTION}

Two types of understanding-errors commonly affect spoken dialog systems: misunderstandings and non-understandings. In a misunderstanding the system constructs an incorrect interpretation of the user's turn, while in a non-understanding the system fails altogether to construct an interpretation. Both types of understandingerrors typically stem from speech recognition problems, and both exert a significant negative impact on the overall quality and success of the interactions [1].

For misunderstandings, detection is the key issue [2]. The number of strategies that can be used to recover is fairly small (e.g. explicit and implicit confirmation) and the tradeoffs between these strategies have been studied and are relatively well understood [3]. In contrast, for non-understandings detection is generally trivial, but the set of strategies that can be used to recover is significantly larger. For instance, the system could ask the user to repeat; it could ask the user to rephrase; it could simply notify the user that a non-understanding happened; it could ignore the non-understanding altogether and try a different dialog plan; it could provide various types of help messages, and so on. The relative tradeoffs between these strategies are less well understood. Moreover, these tradeoffs might be domain- and task-dependent. As a consequence, designing a policy for choosing between different strategies is a non-trivial task. Most spoken dialog systems use a limited number of non-understanding recovery strategies in conjunction with simple heuristic rules for engaging them. A typical example is the so-

\footnotetext{
${ }^{1}$ By recovery strategy we denote a single, one-turn action the system takes to recover from an error (e.g. asking the user to repeat, asking the user to rephrase, etc). By recovery policy we denote a method for choosing between different recovery strategies at runtime.
}

called "three strikes and you're out" approach [4]: repeat the system question after the first non-understanding, provide help after the second one, and transfer the user to a human operator if a third consecutive non-understanding occurs.

In this paper we address the problem of designing a nonunderstanding recovery policy over a large set of non-understanding recovery strategies (9 in our case). We present a supervised, online-learning method for this task. The approach, discussed in detail in Section 2, consists of two steps: first, we construct runtime estimates for the likelihood of success of each individual strategy, together with confidence bounds. Then, we use these estimates to choose between the strategies, and construct a recovery policy.

We implemented and evaluated the proposed approach in a telephone-based spoken dialog system that provides bus route and schedule information in the greater Pittsburgh area. The system learned a non-understanding recovery policy online, throughout a period of 5 weeks. Our experiments indicate that the learned policy led to a $12.5 \%$ relative improvement in the non-understanding recovery rate. Furthermore, the improvement was attained quickly, in only 10 days from the beginning of the learning period.

\section{METHOD}

The starting point for the proposed approach is the intuition that certain non-understanding recovery strategies are more likely to succeed under certain circumstances. For instance, if the source of the non-understanding is an out-of-vocabulary word, asking the user to repeat is less likely to help than asking the user to rephrase. However, if the non-understanding is caused by a transient noise, asking the user to repeat might be a more appropriate course of action. If we could estimate the likelihood of success for each strategy, an optimal policy would be easy to construct: simply pick the strategy with the highest likelihood of success. The method we are proposing works therefore in two steps: first, we use a supervised learning approach to construct predictors for the likelihood of success of each individual recovery strategy. Then, we use these predictors at runtime to select which strategy to engage.

\subsection{Learning Predictors for Strategy Success}

To predict the likelihood of success for each recovery strategy, we use logistic regression models. One separate model is constructed for each strategy. Its goal is to predict whether or not the strategy has successfully recovered, i.e. put the dialog back on track following a given non-understanding. We consider that a strategy has successfully recovered if the following user turn is correctly understood by the system. For training and evaluation purposes, this information is manually annotated. In fact, since the system already 
knows when non-understandings occur, a semi-automatic approach can be used to create the recovery labels: all the non-understandings followed by another non-understanding are automatically labeled as not-recovered; the remaining non-understandings are inspected and labeled by a human annotator. The features (i.e. the dependent variables in the regression model) capture various aspects of the last non-understanding (e.g. the number of words, acoustic, language modeling and goodness-of-parse scores, etc.), as well as information about the current dialog state and about the history of the dialog so far (e.g. number of previous non-understandings, previous recovery actions taken.)

Logistic regression models [5] present a number of advantages over other machine learning techniques in this task. In contrast with other discriminative approaches, logistic regression generally produces well-calibrated class posterior probability scores $[6,7]$. In other words, the model predictions accurately reflect the probability of success (e.g. a strategy will be successful in $x \%$ of the cases when the model predicts that the likelihood of success is $\mathrm{x})$. This is an important property since we plan to use the model scores as probability estimates. Secondly, logistic regression models can automatically provide the confidence intervals for these predictions, a prerequisite for the strategy selection method described in the next subsection. Furthermore, logistic regression is sample efficient. This is another desirable property since we plan to learn one separate model for each strategy and therefore a relatively small number of data-points will be available for training each predictor. Last but not least, logistic regression models can be constructed in a stepwise manner. This allows us to consider a very large number of features; the relevant features will be automatically included in the model.

\subsection{Highest Upper Bound Strategy Selection}

Once we can predict the likelihood of success for each strategy, we are left with choosing the method for selecting between the strategies. Ideally, we should choose the strategy with the highest likelihood of success. However, we are interested in developing an approach in which the system learns a recovery policy on-line, through experimentation. As a result, we are faced with an exploration-exploitation tradeoff. We need to strike a balance between using strategies we know to be successful (exploitation) and gathering more training data for the strategies about which we are still unsure (exploration).

We address this tradeoff by always selecting the strategy that has the highest upper bound on the estimated probability of success. This selection method, also known as the interval estimation, was initially proposed by Kaelbling in [8], and has been shown empirically to perform very well in various explorationexploitation tasks.

Intuitively, by selecting the strategy with the highest upper bound, we either choose a strategy that has a high likelihood of success (Figure 1.a), or we choose a strategy that has a wide confidence interval (Figure 1.b1). In the first case, we are exploiting, in the second we are exploring. After a strategy is engaged, we obtain a new data-point for training the predictor for that strategy. As more and more data becomes available for a strategy, the corresponding confidence interval will shrink. As a result, another strategy will have the highest upper bound, and the system will switch to exploring that strategy (Figure $1 . \mathrm{b} 1 \rightarrow 1 . \mathrm{b} 2$ ).

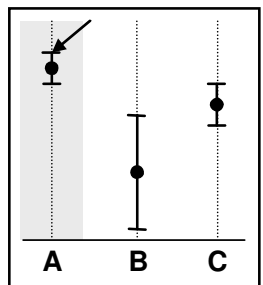

(a)

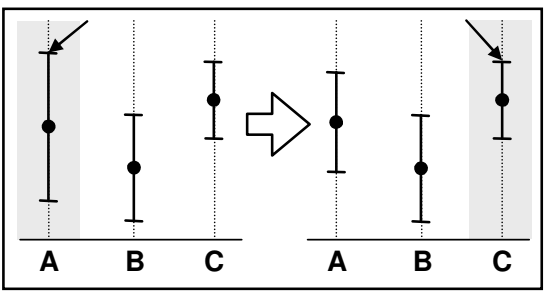

(b.1)

(b.2)
Figure 1. Highest-upper-bound selection between 3 fictitious strategies (A, B and C)

\section{EXPERIMENTAL SETUP}

\subsection{System}

The experiments described below were performed in the context of Let's Go! [9], a telephone-based spoken dialog system which provides access to bus route and schedule information for Pittsburgh Port Authority busses. Since March 2005, the system has been available to the general public via the Port Authority customer service line during non-business hours (i.e. $7 \mathrm{pm}-7 \mathrm{am}$ on weekdays and $6 \mathrm{pm}-8 \mathrm{am}$ on weekends and holidays). Throughout this time, the system has serviced over 23,000 calls. On average, the system receives about 50 calls per night. Given this density of calls, this system provided an excellent basis for our experiment.

\subsection{Strategies}

During the first year of operation, the system used 5 non-understanding recovery strategies in conjunction with a simple heuristic policy (for more details, see [9]). Prior to starting the policy learning experiment, we redesigned the set of non-understanding recovery strategies. The final set is shown in Table 1.

Additionally, we also designed a set of rules to restrict the circumstances under which each strategy can be used. Here are some examples: don't ask the user to repeat more than twice in a row; don't ask the user to give shorter answers unless the number of words is greater than 5; don't ask the user to rephrase if the number of words is below 3; don't give up unless the number of turns is above 30 and the ratio of non-understandings so far above $80 \%$. These rules encapsulate prior expert knowledge. They are used to ensure that the system never uses an unreasonable policy as well as to constrain the search space for the policy learning algorithm. In effect, they implement a heuristic strategy selection policy, which, instead of selecting one strategy, selects a set of valid strategies at each non-understanding. Given these heuristic constraints, the average number of strategies available to the system was 4.2 , with a minimum of 1 and a maximum of 9 .

\subsection{Features}

We identified a large set of features (294) which carry potentially relevant information for predicting the likelihood of successful recovery. Due to space constraints, we only provide a brief outline of the feature set (the full set is available online [10]):

- features describing the current non-understanding: speech recognition features (e.g. acoustic and language model scores, speech rate, signal and noise levels, clipping information), lexical features (e.g. number of words, presence and absence 


\begin{tabular}{|l|l|}
\hline Name & Description \\
\hline \hline HLP & $\begin{array}{l}\text { Give a help message indicates how users might } \\
\text { answer the current system question }\end{array}$ \\
\hline HLP_R & $\begin{array}{l}\text { Same as above, but also tell users that they can } \\
\text { say start-over to restart the dialog }\end{array}$ \\
\hline RP & Repeat the previous system prompt \\
\hline AREP & Ask user to repeat what they said \\
\hline ARPH & Ask user to rephrase what they said \\
\hline MOVE & $\begin{array}{l}\text { Ignore the current non-understanding and back- } \\
\text { off to an alternative dialog plan (this strategy } \\
\text { task-specific and was only available when the } \\
\text { system requested the departure stop; when en- } \\
\text { gaged, the strategy would first request the de- } \\
\text { parture neighborhood, then a departure stop in } \\
\text { that neighborhood) }\end{array}$ \\
\hline ASA & Ask user for a shorter answer \\
\hline SLL & Ask user to speak less loud \\
\hline IT & Give general interaction tips to the user \\
\hline ASO & Ask user if he/she would like to start over \\
\hline GUP & Give up dialogue and hang up \\
\hline
\end{tabular}

Table 1. 11 non-understanding recovery strategies in the Let's Go! Public Bus Information system

of confirmation markers), grammar features (e.g. various goodness-of-parse scores, number of grammar slots), timing information (e.g. barge-ins and timeouts), the non-understanding type (e.g. no-parse vs. rejection);

- features describing the current non-understanding segment: the length of the current non-understanding segment; information about which recovery strategies were already taken in the current non-understanding segment, etc.;

- features describing the current dialog state and the dialog history: we encoded the 22 dialog states with a set of 22 binary variables; additionally, we computed history features such as the ratio of non-understandings, and running averages of confidence scores, goodness-of-parse scores, acoustic and language model scores, etc.

\subsection{Learning}

We started the experiment on March $11^{\text {th }} 2006$. First, we constructed a baseline by running the system for 2 weeks with the new set of 11 recovery strategies. During this time, the system randomly chose a recovery strategy whenever a non-understanding happened, while obeying the set of constraints described in Section 3.2. In effect, the system was using a heuristic, stochastic nonunderstanding recovery policy.

After 2 weeks, on March $26^{\text {th }}, 2006$ we started the online policy learning algorithm. During this learning phase, we excluded the last two strategies shown in Table 1, due to their incompatibility with our local definition of successful recovery. The first excluded strategy (ASO) notifies the user that a non-understanding occurred and asks if the user would like to start over. This generally elicits a yes/no type answer from the user. Although this answer might be correctly understood by the system in most cases, a correct understanding does not really represent a successful recovery from the previous non-understanding. Similarly, when the give up (GUP) strategy is engaged, the system apologizes, asks the user to call during normal business hours, and hangs up. No recovery is therefore possible in this case.
Throughout the learning phase, we retrained the models on a daily basis. Each morning the data from the previous night was semi-automatically labeled with non-understanding recovery information; the models for predicting the likelihood of success for each strategy were retrained and introduced in the system for the following night. We allowed the system to learn in this manner for 5 weeks, until May $5^{\text {th }}$, when we stopped the experiment.

\section{RESULTS}

To evaluate the proposed approach we computed the average nonunderstanding recovery rate (ANRR) throughout the baseline and learning periods. The presence of two extra strategies (ASO and GUP) during the baseline period could confound the results. To make the comparison fair, we excluded all the sessions from the baseline period in which these strategies were engaged (27 out of 524). In fact, this approach artificially inflates the baseline performance of the system. The reason is that, because of the heuristic constraints, the ASO and GUP strategies were only available during sessions with large numbers of non-understandings. ASO was available when the non-understanding ratio was $>50 \%$, GUP when this ratio was $>80 \%$. By eliminating any session that contained one of these strategies we are therefore also eliminating a significant number of unrelated non-understandings, which were not recovered. This artificially inflates the baseline performance. Nevertheless, we can still detect a learning effect.

The resulting daily and weekly averages for the non-understanding recovery rate (ANRR) throughout the baseline and learning periods are illustrated in Figure 2. Despite fairly wide daily fluctuations, a comparison of average recovery performance between the last and first two weeks reveals a statistically significant improvement from $33.6 \%$ to $37.8 \%$ (a $12.5 \%$ relative improvement, $\mathrm{p}=0.0309$ ).

To better understand the learning process we fitted a learning curve to the data, described by the following equation:

$$
A N R R \leftarrow A+B \cdot \frac{e^{C \cdot n+D}}{1+e^{C \cdot n+D}}
$$

The curve describes a temporal learning process ( $n$ is the number of days elapsed) that starts from the baseline $A N N R=A$ and asymptotes at $A N R R=A+B$. The learning rate is captured by the $C$ parameter. The $D$ parameter allows for a shift in the starting point for the learning. The resulting fit is also illustrated in Figure 2. The coefficients were $A=0.3385, B=0.0470, C=0.5566, D=-11.44$. The

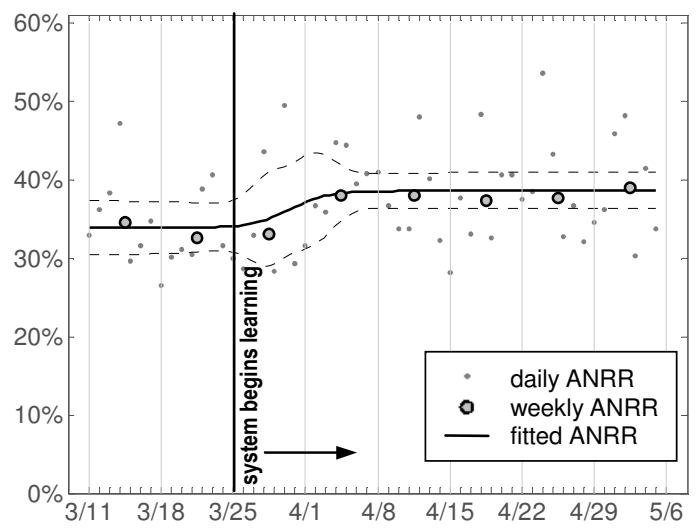

Figure 2. Improvement in average non-understanding recovery rate (ANRR) 


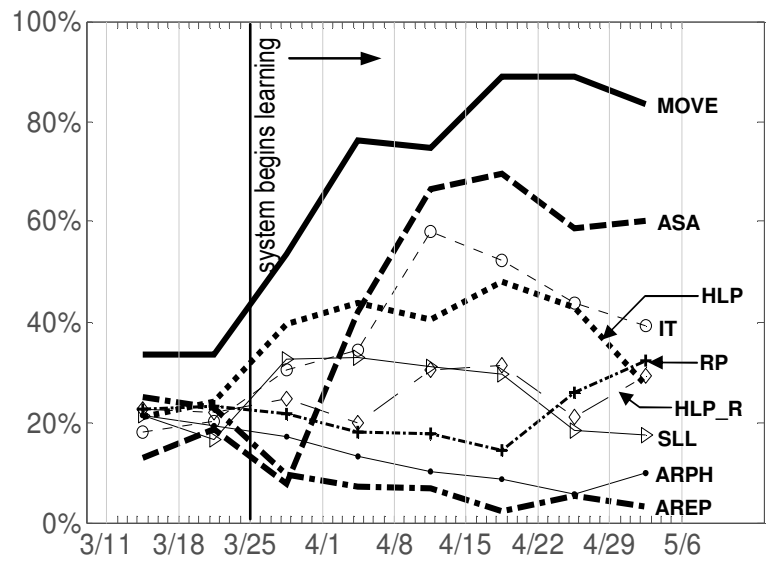

Figure 3. Evolution of invocation percentages for each of the 9 recovery strategies

fitting process recovered our baseline $(A=33.85 \%)$ and indicates that the asymptote $A N R R$ is $38.55 \%(A+B)$. Furthermore, the fitted line also recovered the starting point for the learning (the curve starts moving up after Match $26^{\text {th }}$ ). More interestingly, the curve reveals that the system reached the asymptote performance quickly, in only ten days after the system started learning.

In Figure 3, we illustrate (on a weekly basis) how often each strategy was engaged, as a proportion of the total number of times that the strategy was available (recall that a set of predefined rules constrained when each strategy was available). Before March $26^{\text {th }}$ (i.e. during the baseline phase) the invocation percentages were roughly constant, since the system randomly chose between the strategies available at any given point. The invocation percentages started changing significantly once the learning phase began. Some of the strategies, like backing off to the neighborhood (MOVE) and providing help about what users can say (HLP) were engaged more often than before, while others such as asking the user to repeat (AREP) or rephrase (ARPH) were engaged less often. The strategy which asks for shorter answers was initially engaged less often (we suspect the predictor was unreliable due to small amounts of training data); as more data accumulated, the strategy was pushed up. These trends are in line with previous empirical observations about the general efficiencies of these strategies [1].

We inspected the final versions of the predictors for the likelihood of success of each individual strategy. For three of the strategies - asking the user to repeat (AREP), providing more interaction tips (IT), and ask the user to speak less loud (SLL) - no informative features were found. The models simply predicted the mean training set success rate for each of these strategies. A potential explanation is that the number of training samples collected by the end of the experiment for each of these strategies is still relatively low $(653,273$, and 300 respectively). At the same time, for the strategies that asked the user to provide a shorter answer (ASA, 637 samples), repeating the system prompt (RP, 2532s), and the two help strategies (HLP, 3698s; HLP_R, 989s) the models included 4 or more features. Some of the most informative features were the dialog state indicators: for instance the help strategies were more successful during explicit confirmation states. Additionally, dialog history features were also informative: the likelihood of recovery was generally increased if the dialog went well so far (i.e. if the ratio of non-understandings was low.

\section{CONCLUSION}

We have presented a supervised, online method for learning nonunderstanding recovery policies. Initial experiments with a publicly available spoken dialog system indicate that the proposed method leads to statistically significant improvements in the average non-understanding recovery rate.

We plan to further investigate this proposed approach. We believe that more improvements can be obtained by identifying other relevant features, and further increasing the number of strategies. We also plan to investigate an unsupervised version of this approach. Instead of defining recovery as "next turn is correctly understood by the system", we could define it as "next turn is not another non-understanding", or "next turn has a high confidence score". While less accurate, training labels to match this definition are available automatically to the system, and we suspect that performance improvements could be attained this way.

\section{ACKNOWLEDGMENTS}

We would like to thank Jeff Schneider and Paul Bennett for useful comments and feedback, and the Port Authority of Allegheny County for their help in making the Let's Go! system available to the general public. This work was supported by DARPA CALO grant O3-000211 and NSF grant 0208835. Any opinions, findings and conclusions expressed in this material are those of the authors and do not necessarily reflect the views of the funding agencies.

\section{REFERENCES}

[1] D. Bohus and A. Rudnicky, 2005 - “Sorry I didn't Catch That! - an Investigation of Non-understanding Errors and Recovery Strategies”, in Proceedings of SIGdial-2005, Lisbon, Portugal. [2] M. Walker, J. Wright, I. Langkilde, 2000 - "Using Natural Language Processing and Discourse Features to Identify Understanding Errors in a Spoken Dialogue System”, in Proceedings of ICML-2000, Stanford, CA

[3] E. Krahmer, M. Swerts, M. Theune, and M. Weegels, 2001 "Error Detection in Spoken Human Machine Interaction", in International Journal of Speech Technology, Vol.4, No.1, 19-29 [4] B. Balentine, and D. Morgan, 1999 - "How to Build a Speech Recognition Application: A Style for Telephony Dialogues”, Enterprise Integration Group, ISBN 0-9671278-15.

[5] R. Myers, D. Montgomery and G. Vining, 2001 - "Generalized Linear Models: with Applications to Engineering and the Sciences", Wiley-Interscience, ISBN 0471355739.

[6] I. Cohen, and M. Goldszmidt, 2004 - "Properties and Benefits of Calibrated Classifiers”, in EMCL/PKDD, Pisa, Italy, 2004.

[7] J. Zhang and Y. Yang, 2004 - "Probabilistic Score Estimation with Piecewise Logistic Regression”, in Proceedings of ICML2004, Banff, Alberta, Canada.

[8] L.P. Kaelbling, 1993 - "Learning in Embedded Systems", the MIT Press, Cambridge, MA

[9] A. Raux, D. Bohus, B. Langner, A.W. Black, M. Eskenazi, 2006 - "Doing Research in a Deployed Spoken Dialog System: One Year of Let's Go! Public Experience”, in Proceedings of Interspeech-2006, Pittsburgh, PA.

[10] D. Bohus - www.cs.cmu.edu/ dbohus/research/slt_aux, web page with auxiliary information about learning nonunderstanding recovery policies 